\title{
Análise da Capacidade Para o Trabalho de Agentes Comunitários de Saúde em João Pessoa-PB
}

\author{
Analysis of the Work Ability of Community Health Workers \\ in João Pessoa-PB
}

\author{
Andreia Marinho Barbosa ${ }^{1}$ \\ Dailton Alencar Lucas de Lacerda ${ }^{2}$ \\ Francisco Demóstenes Abrantes Viana ${ }^{3}$
}

\section{RESUMO}

Objetivo: Analisar a Capacidade para o Trabalho (CT) de Agentes Comunitários de Saúde (ACS) de um Distrito Sanitário em João Pessoa-PB. Metodologia: estudo descritivo e transversal de abordagem quantitativa, realizado em João Pessoa-PB, nas 40 Unidades de Saúde da Família pertencentes ao Distrito Sanitário II, com uma amostra de 163 ACS selecionados por conveniência. Os participantes responderam a um questionário semiestruturado, contemplando informações sociodemográficas e o Índice de Capacidade para o Trabalho (ICT), instrumento utilizado para avaliar a CT por meio de sete dimensões. A análise dos dados foi realizada por meio do programa estatístico SPSS 22.0. As variáveis categóricas foram analisadas por meio de frequências absolutas e relativas, utilizando-se o teste do qui-quadrado para estabelecer as relações. O nível de significância adotado foi de $5 \%$. Resultados: a população do estudo foi predominantemente do sexo feminino $(76,7 \%)$, com idade entre 42 e 62 anos $(38,7 \%)$, casada ou com companheiro (a) $(58,3 \%)$, cor da pele parda $(57,1 \%)$, ensino médio completo $(72,4 \%)$, renda familiar de até 2 salários mínimos $(51,0 \%)$, com capacidade boa $(38,7 \%)$ e moderada $(37,4 \%)$ para o trabalho. A CT apresentou associação estatisticamente significativa com cada uma das sete dimensões do ICT $(p<0,05)$. As doenças mais frequentemente informadas foram: lesões musculoesqueléticas $(55,2 \%)$, doenças musculoesqueléticas $(44,8 \%)$, obesidade $(34,4 \%)$, hipertensão arterial $(27,0 \%)$ e distúrbio emocional leve (26,4\%). Conclusão: os ACS desse estudo apresentaram boa capacidade de trabalho de acordo com o ICT.

DESCRITORES: Agentes Comunitários de Saúde; Saúde do Trabalhador; Estratégia Saúde da Família; Avaliação da Capacidade de Trabalho.

\begin{abstract}
Objective: analyze the Work Ability (CT) of Community Health Workers (ACS) of a Sanitary District in João Pessoa-PB. Methodology: Descriptive and transversal study of quantitative approach, conducted in João Pessoa-PB, in the 40 Family Health Units belonging to the Sanitary District II, with a sample of 163 ACS selected by convenience. Participants answered a semi-structured questionnaire, including socio-demographic information and the Work Ability Index (ICT), instrument used to evaluate CT through seven dimensions. Data analysis was performed using the statistical program SPSS Statistics, version 22.0. The categorical variables were analyzed through absolute and relative frequencies, using the chi-square test to establish relations. The significance level was $5 \%$. Results: the study population was predominantly female $(76,7 \%)$, aged $42-62$ years $(38,7 \%)$, married or with partner $(58,3 \%)$, brown skin color $(57,1 \%)$, complete high school $(72,4 \%)$, family income of up to 2 minimum wages $(51,0 \%)$, with good capacity $(38,7 \%)$ and moderate capacity $(37,4 \%)$ for work. The CT showed a statistically significant association with each of the seven dimensions of the ICT $(p<0,05)$. The most frequently reported diseases were: musculoskeletal disorders $(55,2 \%)$, musculoskeletal diseases $(44,8 \%)$, obesity $(34,4 \%)$, hypertension $(27,0 \%)$ and mild emotional disturbance $(26,4 \%)$ Conclusion: the ACS of this study had good working capacity according to the ICT.
\end{abstract}

DESCRIPTORS: Community Health Workers; Occupational Health; Family Health Strategy; Work Capacity Evaluation.

\footnotetext{
1- Mestranda no Programa de Pós-graduação em Saúde da Família. Universidade Federal da Paraíba (UFPB). João Pessoa. Paraíba. Brasil.

2- Professor no Departamento de Fisioterapia da Universidade Federal da Paraíba (UFPB). João Pessoa. Paraíba. Brasil.
}

3- Enfermeiro. São João do Rio do Peixe. Paraíba. Brasil. 
A Capacidade para o Trabalho (CT) é a disposição que o indivíduo tem para executar suas atividades laborais em função das exigências ocupacionais, levando em consideração seu estado físico e mental, sendo influenciada por fatores ambientais e condições de trabalho, além do estilo de vida ${ }^{1,2}$.

O impacto que o trabalho exerce sobre a saúde tem sido investigado com regularidade em diversas categorias profissionais ${ }^{1,3-6}$. No âmbito da saúde, especificamente na Atenção Básica $(A B)$, importantes fragilidades de ordem estrutural, política e cultural, impactam negativamente nas cargas de trabalho dos profissionais de saúde, como a falta de entendimento dos gestores e usuários quanto à organização da Estratégia de Saúde da Família (ESF) ${ }^{7}$.

O Agente Comunitário de Saúde (ACS) é um profissional de saúde vinculado a ESF, que exerce suas funções de acordo com atribuições definidas na Portaria $n^{\circ}$. 2.436/2017, na qual consta que o ACS deve exercer ações que busquem a integração entre a equipe de saúde e a população adscrita à Unidade Básica de Saúde (UBS), acompanhar todas as famílias e indivíduos sob sua responsabilidade, desenvolver ações educativas nos domicílios e na comunidade e manter a equipe informada sobre a situação de saúde dos atendidos ${ }^{8}$.

Esses profissionais em seu cotidiano enfrentam um dilema entre o excesso de demandas no processo de trabalho e pressão da gestão/ comunidade. Como consequência, convivem com cobranças que geram sobrecargas físicas e mentais, traduzidas por estresse, ansiedade, tensão muscular, insônia, sofrimento psíquico, entre outras ${ }^{9-11}$.

Apesar disso, ainda existem poucos estudos na literatura que se propõem a analisar a CT desses profissionais de saúde. Destaca-se um realizado em Uberaba-MG ${ }^{12}$, que avaliou a associação entre CT e sintomas osteomusculares em ACS, verificando que 55,32\% apresentaram capacidade inadequada para o trabalho e $93,62 \%$ expôs sintomas osteomusculares nos últimos 12 meses.

O Sistema Único de Saúde (SUS) é o responsável pela execução de ações que visem à vigilância em saúde do trabalhador, de modo a promover, proteger, recuperar e reabilitar a saúde dos trabalhadores submetidos aos riscos e agravos advindos das condições de trabalho ${ }^{13}$. Por isso, contribuir com estudos que buscam avaliar a capacidade que os trabalhadores apresentam para desenvolver suas atividades laborais devem ser incentivados, especialmente no campo da saúde, no qual esses profissionais também devem estar atentos a sua saúde individual e sua relação com o ambiente de trabalho.

Diante do exposto, esse trabalho objetivou analisar a capacidade para o trabalho dos ACS de um Distrito Sanitário em João Pessoa-PB.

\section{METODOLOGIA}

Trata-se de um estudo descritivo e transversal com abordagem quantitativa, desenvolvido no Município de João Pessoa-PB, especificamente no Distrito Sanitário II (DS II) em suas 40 Unidades de Saúde da Família (USF).

Os dados foram coletados no período de abril a junho de 2014, por uma equipe constituída de três acadêmicas previamente treinadas. Uma relação com o nome e endereço atualizado das USF do DS II foi fornecida pela Gerência de Educação na Saúde (GES), da Diretoria de Gestão e Trabalho e Educação na Saúde, da Secretaria Municipal de Saúde de João Pessoa-PB (SMSJP). A partir de então, realizou-se o contato com as Unidades e agendou-se um dia de reunião de equipe para conversar com os ACS, apresentandoIhes a pesquisa.

A população da pesquisa constituiu todos os ACS vinculados ao DS II. A seleção da amostra foi realizada por conveniência onde, de um universo de 279 ACS, 59,5\% ( $n=166)$ estavam presentes nas USFs no momento da coleta de dados, e $58,4 \%(n=163)$ concordaram em participar da pesquisa assinando o Termo de Consentimento Livre e Esclarecido (TCLE). Estes responderam individualmente a um questionário semiestruturado, contemplando dados sociodemográficos (sexo, idade, estado civil, escolaridade e renda familiar), e o Índice de Capacidade para o Trabalho (ICT).

O ICT é um instrumento composto por dez questões sintetizadas em sete dimensões, que permitem avaliar a Capacidade para o Trabalho (CT) por meio da percepção do próprio trabalhador (Quadro 1). Os resultados obtidos a partir dessas dimensões fornecem uma medida da CT que varia de 07 a 49 pontos: CT baixa (07 a 27 pontos); CT moderada (28 a 36 pontos); CT boa (37 a 43 pontos); e CT ótima (44 a 49 pontos). Para cada classificação, indica-se também a necessidade de medidas que visem: restaurá-la; melhorá-la; apoia-lá; e mantê-la, respectivamente ${ }^{2}$. 


\begin{tabular}{|c|c|c|}
\hline Item & $\begin{array}{c}\mathbf{N}^{0} \text { de } \\
\text { questões }\end{array}$ & Escore das respostas \\
\hline $\begin{array}{lll}\text { 1. Capacidade atual para } & \circ \\
\text { trabalho comparada } & \text { com a } \\
\text { melhor de toda a vida } & & \\
\end{array}$ & 1 & $\begin{array}{llllll}\text { De } & 0 & \text { a } & 10 & \text { pontos } & \text { (valor assinalado no } \\
\text { questionário) } & & & & \end{array}$ \\
\hline $\begin{array}{l}\text { 2. Capacidade para o trabalho } \\
\text { em relação as exigências do } \\
\text { trabalho }\end{array}$ & 2 & $\begin{array}{l}\text { De } 0 \text { a } 10 \text { pontos (considera a natureza do } \\
\text { trabalho física e/ou mental) }\end{array}$ \\
\hline $\begin{array}{l}\text { 3. Número de doenças atuais } \\
\text { diagnosticadas por médico }\end{array}$ & $\begin{array}{l}1 \text { (lista de } 51 \\
\text { doenças) }\end{array}$ & $\begin{array}{l}\text { De } 0 \text { a } 7 \text { pontos (pelo menos } 5 \text { doenças }=1 \\
\text { ponto; } 4 \text { doenças }=2 \text { pontos; } 3 \text { doenças }=3 \\
\text { pontos; } 2 \text { doenças }=4 \text { pontos; } 1 \text { doença }=5 \\
\text { pontos; nenhuma doença }=7 \text { pontos) }\end{array}$ \\
\hline $\begin{array}{l}\text { 4. Perda estimada para } \circ \\
\text { trabalho por causa de doenças }\end{array}$ & 1 & $\begin{array}{l}\text { De } 1 \text { a } 6 \text { pontos (valor circulado no questionário; } \\
\text { o pior valor será o escolhido) }\end{array}$ \\
\hline $\begin{array}{l}\text { 5. Faltas ao trabalho (dias) por } \\
\text { doenças nos últimos } 12 \text { meses }\end{array}$ & 1 & $\begin{array}{l}\text { De } 1 \text { a } 5 \text { pontos (inversamente ao valor } \\
\text { apontado). Para nenhum dia } 5 \text { pontos e no } \\
\text { mínimo } 100 \text { dias } 1 \text { ponto }\end{array}$ \\
\hline $\begin{array}{l}\text { 6. Prognóstico } \\
\text { capacidade para } \\
\text { daqui a } 2 \text { anos }\end{array}$ & 1 & $\begin{array}{l}1,4 \text { ou } 7 \text { pontos (incapaz, incerto e capaz, } \\
\text { respectivamente) }\end{array}$ \\
\hline $\begin{array}{l}\text { 7. Capacidade de apreciar a } \\
\text { vida, de se sentir alerta e da } \\
\text { esperança no futuro. }\end{array}$ & 3 & $\begin{array}{l}\text { Cada questão varia de } 0 \text { a } 4 \text { (os valores das } \\
\text { questões são somados e contados: } 0-3=1 \text { ponto; } \\
4-6=2 \text { pontos; } 7-9=3 \text { pontos; } 10-12=4 \text { pontos) }\end{array}$ \\
\hline
\end{tabular}

Os dados coletados foram transcritos para meio digital com a utilização da planilha eletrônica do programa Microsoft Excel $®$ versão 2010, e posteriormente exportados para o programa estatístico SPSS 22.0, no qual as variáveis categóricas foram analisadas por meio de frequências absolutas e relativas. Para estabelecer as relações entre as variáveis utilizou-se o teste do qui-quadrado. O nível de significância adotado foi de $5 \%$.

O estudo foi submetido ao Comitê de Ética em Pesquisa do Centro de Ciências da Saúde da Universidade Federal da Paraíba e aprovado de acordo com o Protocolo n ${ }^{\circ}$. 0104, de 20/03/2014. Todos os princípios éticos dispostos na Resolução $n^{\circ}$. 466/2012 do Conselho Nacional de Saúde (CNS) foram respeitados. -

\section{RESULTADOS}

Apopulação do estudo foi predominantemente do sexo feminino $(76,7 \%)$, com idade entre 42 e 62 anos (38,7\%), casada ou com companheiro(a) $(58,3 \%)$, cor da pele parda $(57,1 \%)$, ensino médio completo $(72,4 \%)$ e renda familiar de até 2 salários mínimos $(51,0 \%)$.
O ICT identificou que a maior parte dos ACS apresentaram capacidade boa $(38,7 \%)$ e moderada $(37,4 \%)$, seguidos por capacidade ótima $(17,8 \%)$ e baixa $(6,1 \%)$ para o trabalho. As variáveis sociodemográficas não apresentaram relação estatisticamente significativa com o ICT (Tabela 1).

Quanto a distribuição dos ACS segundo as dimensões que compõem o ICT encontrou-se os seguintes resultados: na "capacidade para o trabalho atual comparada com a melhor de toda a vida" $68,1 \%$ dos trabalhadores alcançaram as três pontuações superiores; na "capacidade para o trabalho em relação às exigências do trabalho" $68,7 \%$ dos trabalhadores alcançaram as três pontuações superiores; no "número atual de doenças diagnosticadas pelo médico" $39,3 \%$ dos trabalhadores referiram presença de pelo menos 5 doenças e 6,2\% informaram não apresentar doenças; na "perda estimada para o trabalho devido às doenças" $46,5 \%$ dos trabalhadores informaram que não têm nenhum impedimento/ doença para realizar seu trabalho; nas "faltas ao trabalho por doenças nos últimos 12 meses" $37,5 \%$ dos trabalhadores negaram afastamento 
Tabela 1. Capacidade para o trabalho segundo as variáveis sociodemográficas dos ACS vinculados as ESF do Distrito Sanitário II, João Pessoa - PB, 2014.

\begin{tabular}{|c|c|c|c|c|c|c|c|c|c|c|}
\hline \multirow{3}{*}{ Variável } & \multicolumn{10}{|c|}{ Índice } \\
\hline & \multirow{2}{*}{$\begin{array}{c}\text { Total } \\
\mathrm{n}\end{array}$} & \multicolumn{2}{|c|}{ Baixa } & \multicolumn{2}{|c|}{ Moderada } & \multicolumn{2}{|c|}{ Boa } & \multicolumn{2}{|c|}{ Ótima } & \multirow{2}{*}{$\begin{array}{l}\text { Valor } \\
\text { de p* }\end{array}$} \\
\hline & & $\mathrm{n}$ & $\%$ & $\mathbf{n}$ & $\%$ & $\mathrm{n}$ & $\%$ & $\mathrm{n}$ & $\%$ & \\
\hline Sexo & & & & & & & & & & 0,072 \\
\hline Feminino & 125 & 9 & 5,5 & 49 & 30,0 & 50 & 30,7 & 17 & 10,4 & \\
\hline Masculino & 38 & 1 & 0,6 & 12 & 7,4 & 13 & 8,0 & 12 & 7,4 & \\
\hline Idade & & & & & & & & & & 0,783 \\
\hline 25 - 33 anos & 50 & 5 & 3,1 & 17 & 10,4 & 19 & 11,7 & 9 & 5,5 & \\
\hline $34-41$ anos & 50 & 2 & 1,2 & 22 & 13,5 & 17 & 10,4 & 9 & 5,5 & \\
\hline $42-62$ anos & 63 & 3 & 1,8 & 22 & 13,5 & 27 & 16,6 & 11 & 6,7 & \\
\hline Estado civil & & & & & & & & & & 0,664 \\
\hline Solteiro(a) & 51 & 4 & 2,4 & 16 & 9,8 & 23 & 14,1 & 8 & 4,9 & \\
\hline $\begin{array}{l}\text { Casado(a)/Mora com } \\
\text { companheiro(a) }\end{array}$ & 95 & 6 & 3,7 & 36 & 22,0 & 35 & 21,5 & 18 & 11,0 & \\
\hline $\begin{array}{l}\text { Separado/Divorciado/ } \\
\text { Viúvo }\end{array}$ & 17 & 0 & 0,0 & 9 & 5,5 & 5 & 3,1 & 3 & 1,8 & \\
\hline Cor da pele & & & & & & & & & & 0,511 \\
\hline Branca & 38 & 2 & 1,2 & 10 & 6,1 & 19 & 11,7 & 7 & 4,3 & \\
\hline Parda & 93 & 7 & 4,3 & 40 & 24,6 & 28 & 17,2 & 18 & 11,0 & \\
\hline Preto & 29 & 1 & 0,6 & 10 & 6,1 & 14 & 8,6 & 4 & 2,4 & \\
\hline Outra & 3 & 0 & 0,0 & 1 & 0,6 & 2 & 1,2 & 0 & 0,0 & \\
\hline Escolaridade & & & & & & & & & & 0,557 \\
\hline Fund. incompleto & 1 & 0 & 0,0 & 1 & 0,6 & 0 & 0,0 & 0 & 0,0 & \\
\hline Fund. completo & 2 & 0 & 0,0 & 1 & 0,6 & 1 & 0,6 & 0 & 0,0 & \\
\hline Médio completo & 118 & 6 & 3,7 & 41 & 25,1 & 45 & 27,6 & 26 & 16,0 & \\
\hline Superior completo & 42 & 4 & 2,4 & 18 & 11,0 & 17 & 10,4 & 3 & 1,8 & \\
\hline Renda Familiar ** & & & & & & & & & & 0,559 \\
\hline Até 2 salários mínimos & 83 & 5 & 3,1 & 26 & 15,9 & 37 & 22,7 & 15 & 9,2 & \\
\hline 2-5 salários mínimos & 66 & 5 & 3,1 & 29 & 17,8 & 20 & 12,3 & 12 & 7,4 & \\
\hline > 5 salários mínimos & 14 & 0 & 0,0 & 6 & 3,7 & 6 & 3,7 & 2 & 1,2 & \\
\hline Total & 163 & 10 & 6,1 & 61 & 37,4 & 63 & 38,7 & 29 & 17,8 & \\
\hline
\end{tabular}

*Teste do qui-quadrado $(p<0,05)$.

*** No período de coleta de dados o valor vigente do salário mínimo era de $\mathrm{R} \$ 724,00$. 
do trabalho por doenças nos últimos 12 meses e $40,5 \%$ referiram que ficaram afastados até 9 dias; no "prognóstico próprio sobre a capacidade para o trabalho" $74,8 \%$ dos trabalhadores consideraram bastante provável que em dois anos sejam capazes de realizar o trabalho atual; e nos "recursos mentais" $57,0 \%$ dos trabalhadores alcançaram a pontuação mais elevada considerando sua capacidade para apreciar as atividades diárias, percepção de estar ativo e alerta, e sentimento de esperança para o futuro (Tabela 2).

ACT apresentou associação estatisticamente significativa com cada uma das sete dimensões do ICT $(p<0,05)$, ou seja, quanto mais alta a pontuação na dimensão do ICT, maior a capacidade para o trabalho dos entrevistados.

As doenças mais frequentemente informadas pelos ACS foram distribuídas conforme mostra a Tabela 3. Quanto à subdivisão das lesões musculoesqueléticas citadas por eles, os resultados constituíram em: nas costas $(28,8 \%)$; pernas/pés (24,5\%); braços/mãos (16,0\%); outras partes do corpo $(9,2 \%)$. No tocante às doenças musculoesqueléticas: na parte superior das costas $(24,5 \%)$; dor nas costas que se irradia para a perna $(14,1 \%)$; na parte inferior das costas $(13,5 \%)$; afeta os membros - braços/pernas (9,8\%); artrite reumatoide $(8,6 \%)$; outras $(6,7 \%)$.

Tabela 2. Distribuição dos ACS segundo pontuação das dimensões do ICT. Distrito Sanitário II, João Pessoa - PB, 2014.

\begin{tabular}{|c|c|c|c|c|c|c|c|c|c|c|c|c|}
\hline \multirow[t]{2}{*}{$\begin{array}{c}\text { Dimensão do } \\
\text { ICT }\end{array}$} & \multicolumn{11}{|c|}{ Pontuação n (\%) } & \multirow[t]{2}{*}{$\begin{array}{l}\text { Total } \\
\text { n (\%) }\end{array}$} \\
\hline & 0 & 1 & 2 & 3 & 4 & 5 & 6 & 7 & 8 & 9 & 10 & \\
\hline $\begin{array}{l}\text { CT atual } \\
\text { comparada } \\
\text { com a melhor } \\
\text { de toda a vida }\end{array}$ & $\begin{array}{c}1 \\
(0,6)\end{array}$ & $\begin{array}{c}0 \\
(0,0)\end{array}$ & $\begin{array}{c}0 \\
(0,0)\end{array}$ & $\begin{array}{c}0 \\
(0,0)\end{array}$ & $\begin{array}{c}3 \\
(1,8)\end{array}$ & $\begin{array}{c}9 \\
(5,5)\end{array}$ & $\begin{array}{c}10 \\
(6,2)\end{array}$ & $\begin{array}{c}29 \\
(17,8)\end{array} \mid$ & $\begin{array}{c}49 \\
(30,1)\end{array}$ & $\begin{array}{c}31 \\
(19,0)\end{array}$ & $\begin{array}{c}31 \\
(19,0)\end{array}$ & $\begin{array}{c}163 \\
(100)\end{array}$ \\
\hline $\begin{array}{l}\text { CT em relação } \\
\text { às exigências } \\
\text { do trabalho }\end{array}$ & $\begin{array}{c}0 \\
(0,0)\end{array}$ & $\begin{array}{c}0 \\
(0,0)\end{array}$ & $\begin{array}{c}0 \\
(0,0)\end{array}$ & $\begin{array}{c}0 \\
(0,0)\end{array}$ & $\begin{array}{c}3 \\
(1,8)\end{array}$ & $\begin{array}{c}6 \\
(3,7)\end{array}$ & $\begin{array}{c}13 \\
(8,0)\end{array}$ & $\begin{array}{c}29 \\
(17,8)\end{array}$ & $\begin{array}{c}53 \\
(32,5)\end{array}$ & $\begin{array}{c}34 \\
(20,9)\end{array}$ & $\begin{array}{c}25 \\
(15,3)\end{array}$ & $\begin{array}{c}163 \\
(100)\end{array}$ \\
\hline $\begin{array}{l}\text { Número atual } \\
\text { de doenças } \\
\text { diagnosticadas } \\
\text { por médico }\end{array}$ & & $\begin{array}{c}64 \\
(39,3)\end{array}$ & $\begin{array}{c}25 \\
(15,3)\end{array}$ & $\begin{array}{c}22 \\
(13,5)\end{array}$ & $\begin{array}{c}18 \\
(11,0)\end{array}$ & $\begin{array}{c}24 \\
(14,7)\end{array}$ & & $\begin{array}{c}10 \\
(6,2)\end{array}$ & & & & $\begin{array}{c}163 \\
(100)\end{array}$ \\
\hline $\begin{array}{l}\text { Perda } \\
\text { estimada para } \\
\text { o trabalho } \\
\text { devido às } \\
\text { doenças }\end{array}$ & & $\begin{array}{c}0 \\
(0,0)\end{array}$ & $\begin{array}{c}7 \\
(4,3)\end{array}$ & $\begin{array}{c}12 \\
(7,4)\end{array}$ & $\begin{array}{c}41 \\
(25,2)\end{array}$ & $\begin{array}{c}27 \\
(16,6)\end{array}$ & $\begin{array}{c}76 \\
(46,5)\end{array}$ & & & & & $\begin{array}{c}163 \\
(100)\end{array}$ \\
\hline $\begin{array}{l}\text { Faltas ao } \\
\text { trabalho por } \\
\text { doenças no } \\
\text { último ano }\end{array}$ & & $\begin{array}{c}11 \\
(6,7)\end{array}$ & $\begin{array}{c}11 \\
(6,7)\end{array}$ & $\begin{array}{c}14 \\
(8,6)\end{array}$ & $\begin{array}{c}66 \\
(40,5)\end{array}$ & $\begin{array}{c}61 \\
(37,5)\end{array}$ & & & & & & $\begin{array}{c}163 \\
(100)\end{array}$ \\
\hline $\begin{array}{l}\text { Prognóstico } \\
\text { próprio sobre a } \\
\text { CT daqui a } \\
\text { dois anos }\end{array}$ & & $\begin{array}{c}10 \\
(6,2)\end{array}$ & & & $\begin{array}{c}31 \\
(19,0)\end{array}$ & & & $\begin{array}{c}122 \\
(74,8)\end{array}$ & & & & $\begin{array}{c}163 \\
(100)\end{array}$ \\
\hline $\begin{array}{l}\text { Recursos } \\
\text { mentais }\end{array}$ & & $\begin{array}{c}0 \\
(0,0)\end{array}$ & $\begin{array}{c}9 \\
(5,5)\end{array}$ & $\begin{array}{c}61 \\
(37,5)\end{array}$ & $\begin{array}{c}93 \\
(57,0)\end{array}$ & & & & & & & $\begin{array}{c}163 \\
(100)\end{array}$ \\
\hline
\end{tabular}

Obs.: os espaços em branco são devido às pontuações não existirem nas respectivas dimensões do ICT. 
Tabela 3. Prevalência de lesões/doenças nos ACS, vinculados a ESF, do Distrito Sanitário II, João Pessoa - PB, 2014.

\begin{tabular}{l|c|c}
\hline Doenças & Casos & Prevalência \\
\hline Lesões musculoesqueléticas & 90 & 55,2 \\
Doenças musculoesqueléticas & 73 & 44,8 \\
Obesidade & 56 & 34,4 \\
Hipertensão Arterial & 44 & 27,0 \\
Distúrbio emocional leve & 43 & 26,4 \\
Alergias & 39 & 23,9 \\
Gastrite gástrica ou duodenal & 33 & 20,2 \\
Sinusite crônica & 25 & 15,3 \\
Infecção no trato respiratório & 22 & 13,5 \\
Anemia & 22 & 13,5 \\
Doença ou lesão na visão & 16 & 9,8 \\
Outras doenças neurológicas & 16 & 9,8 \\
Problema ou diminuição da audição & 14 & 8,6 \\
Doença nos rins & 14 & 8,6 \\
Outra doença de pele & 14 & 8,6 \\
Outras doenças respiratórias & 12 & 7,3 \\
Diabetes & 11 & 6,7 \\
Dislipidemia & 9 & 5,5 \\
Outras doenças cardiovasculares & 8 & 4,9 \\
Bócio ou outra doença na tireoide & 8 & 4,9 \\
Outro problema ou doença & 8 & 4,9 \\
Doença coronariana & 7 & 4,3 \\
Asma & 7 & 4,3 \\
Distúrbio emocional severo & 7 & 4,3 \\
Bronquite crônica & 6 & 3,7 \\
Pedras ou doenças na vesícula biliar & 5 & 3,1 \\
Outra doença geniturinária & 5 & 3,1 \\
Doença no pâncreas ou fígado & 4 & 2,5 \\
Outras doenças digestivas & 4 & 2,5 \\
Úlcera gástrica ou duodenal & 3 & 1,8 \\
Infecção nas vias urinárias & 3 & 1,8 \\
Doença nos genitais e aparelho reprodutor & 3 & 1,8 \\
Tumor benigno & 3 & 1,8 \\
Outra doença endócrina ou metabólica & 3 & 1,8 \\
Tuberculose & 2 & 1,2 \\
Tumor maligno & 2 & 1,2 \\
\hline & & \\
\hline
\end{tabular}

\section{DISCUSSÃO}

A predominância do sexo feminino entre ACS pode estar intimamente ligado ao papel de cuidador que a mulher desempenha na sociedade ${ }^{14}$ e a resistência ao agente do sexo masculino por parte da comunidade ${ }^{15}$. A faixa etária que mais concentrou agentes, também foi observada em estudos realizados com ACS em Porto Alegre-RS ${ }^{14}$ e Uberlândia-MG ${ }^{9}$.
Quanto ao grau de escolaridade, a Lei $\mathrm{n}^{\circ}$. $13.595 / 2018$ que alterou a Lei $n^{\circ} .11 .350 / 2006$ que regulamenta a profissão, tornando exigência para o cargo ter a conclusão do Ensino Médio - antes fundamental - e o curso de formação inicial ${ }^{16}$. Os resultados dessa pesquisa vão de encontro a esta nova redação, pois os ACS em sua maioria possuem ensino médio completo. Isso repercute como estratégia positiva que demonstra que quanto 
maior o grau de escolaridade, mais possibilidades terá o agente de adquirir novos conhecimentos que nortearão suas práticas em saúde ${ }^{14}$.

$\mathrm{Na}$ literatura, constata-se a existência de insatisfação dos ACS em relação a sua renda quando comparada a de outros profissionais da saúde ${ }^{17,18}$, o que pode gerar desmotivação e sentimento de desvalorização, influenciando negativamente na sua qualidade de vida ${ }^{19,20}$.

Os achados encontrados quanto à CT foram semelhantes aos de estudos conduzidos com trabalhadores de enfermagem ${ }^{1}$ e higiene/limpeza ${ }^{6}$ de hospitais universitários. No primeiro estudo a prevalência da CT boa/ótima foi de 56,7\%; no segundo essa mesma faixa alcançou um nível de $69,4 \%$. Esse fato, porém, pode estar atrelado ao "efeito do trabalhador sadio" sobre a população estudada, um fenômeno observado em estudos de doenças ocupacionais, onde trabalhadores geralmente exibem melhores indicadores de morbimortalidade quando comparados à população em geral devido ao fato dos portadores de doenças graves ou incapacitados normalmente estarem excluídos da força de trabalho ativa ${ }^{21,22}$. Como é o caso desta pesquisa, onde $40,5 \%$ dos ACS não estavam presentes no momento da coleta de dados devido a problemas de saúde, férias, licença-maternidade, entre outros.

Nesse estudo, mesmo a faixa etária mais velha (42-62 anos) ser a que mais concentrou ACS, estes ainda mostraram uma boa CT (16,6\%). Este resultado divergiu de um estudo realizado com ACS em Uberaba-MG ${ }^{12}$, onde aqueles que estavam com capacidade inadequada apresentavam uma média de idade maior $(39,35 \pm 13,39)$, mesmo sem correlação significativa.

$\mathrm{Na}$ análise individual dos componentes do ICT, mais da metade dos participantes indicaram adequada CT atual comparada a melhor de toda a vida (acima de 7 na escala), corroborando com uma pesquisa realizada com enfermeiros de um Hospital Universitário no Rio Grande do Sul, que destacou na capacidade para o trabalho atual, uma elevada média na autoavaliação $(8,6 \pm 1,1)^{23}$.

Os resultados dos demais componentes do ICT encontrados foram semelhantes ao de outro estudo realizado com trabalhadores de enfermagem ${ }^{24}$ quanto às exigências físicas (44,4\%) e mentais $(63,5 \%)$; perda estimada para o trabalho devido às doenças: não há impedimento $(33,3 \%)$; ausência no trabalho por problemas de saúde nos últimos 12 meses: nenhum dia (42,9\%) e até 9 dias (25,4\%); prognóstico próprio sobre a capacidade para o trabalho: $65,1 \%$ alegaram ser bastante provável que em dois anos sejam capazes de fazer o trabalho atual; e na satisfação com as atividades diárias, 52,4\% estavam quase sempre satisfeitos. Os principais problemas de saúde relatados pelos trabalhadores das ESFs da Macrorregião Nordeste de Minas Gerais referem-se aos sistemas circulatório, musculoesquelético, respiratório, endócrino-metabólico e nervoso, com ênfase, respectivamente, de cada grupo: hipertensão $(85,7 \%)$; dor lombar/coluna $(48,7 \%)$; alergia respiratória $(62,5 \%)$; diabetes $(37,8 \%)$ e cefaleia $(71,0 \%)^{25}$. Tais achados são semelhantes aos encontrados nesta pesquisa, exceto pela obesidade e o distúrbio emocional leve que estão entre as cinco doenças mais prevalentes entre os ACS.

A obesidade como doença endócrina, com maior prevalência, alerta para a atual transição nutricional enfrentada pela população brasileira. Com o aumento do sobrepeso e da obesidade, as pessoas estão mais sujeitas a outros tipos de adoecimento, entre eles, as doenças cardiovasculares, diabetes e certos tipos de câncer ${ }^{26}$, além de que o excesso de peso é tido como fator de risco para a perda da $\mathrm{CT}^{27}$.

Os achados desse trabalho também constatam a presença de sofrimento psíquico entre esses trabalhadores. Esse agravo é observado na literatura quando se trata da saúde ocupacional dos profissionais de saúde em geral ${ }^{28}$ e, ao envolver ACS, podem ser potencializados pela alta demanda psicológica das tarefas, vivência com agressões e insatisfação com as relações pessoais $^{22}$.

No tocante as doenças diagnosticadas por médico, destacaram-se as musculoesqueléticas assim como em outros estudos realizados com a classe de trabalhadores de enfermagem hospitalar ${ }^{23,29}$ e $\mathrm{ACS}^{12}$. Neste último, observou-se um alto número de ACS apresentando sintomas osteomusculares $(82,98 \%$ nos últimos sete dias e $93,62 \%$ nos últimos 12 meses) ${ }^{12}$.

Outra análise sobre os distúrbios musculoesqueléticos entre os trabalhadores da saúde identificou os ACS como a $3^{a}$ categoria que apresentava maiores prevalências desses distúrbios (59,6\%), sendo superados apenas pelos profissionais técnicos de nível superior $(61,4 \%)$, e dentistas e técnicos de odontologia $(61 \%)^{30}$.

Pesquisas que avaliam as relações entre trabalho e saúde dos ACS ainda são escassas, entretanto pelas suas próprias características de trabalho, como deslocamentos diários e longos percursos para acessar os domicílios dos usuários, deixa-os expostos a condições que propiciam o aparecimento de distúrbios musculoesqueléticos ${ }^{30}$

Novas investigações que se proponham a avaliar a capacidade para o trabalho e sua influência na saúde dos ACS são recomendadas, 
até porque pela limitação do estudo transversal não é possível identificar nessa pesquisa a sequência de fator e efeito e estabelecer relações causais entre as variáveis.

\section{REFERÊNCIAS}

1. Magnago TSBS, Prochnow A, Urbanetto JS, Greco PBT, Beltrame M, LUZ EMF. Relationship between work ability in nursing and minor psychological disorders. Texto \& contexto enferm. 2015; 24(2):362-370.

2. Tuomi K, IImarinen J, Jahkola A, Katajarinne L, Tulkki A. Índice de capacidade para o trabalho. São Carlos: EduFSCar; 2005.

3. Vidotti HGM, Coelho VHM, Bertoncello D, Walsh IAP. Qualidade de vida e capacidade para o trabalho de bombeiros. Fisioter. pesqui. 2015; 22(3):231-238

4. Moura AL, Reis LM, Vannuchi MTO, Haddad MDCL, Domansky RC. Capacidade para o trabalho de funcionários da prefeitura de um campus universitário público. Rev. eletrônica enferm. 2013; 15(1):130-7.

5. Costa CSN, Freitas EG, Mendonça LCS, Alem MER, Coury HJCG. Capacidade para o trabalho e qualidade de vida de trabalhadores industriais. Ciênc. saúde coletiva. 2012; 17(6):1635-1642.

6. Silva LG, Haddad MCL, Domansky RC, Vituri DW. Capacidade para o trabalho entre trabalhadores de higiene e limpeza de um hospital universitário público. Rev. eletrônica enferm. 2010; 12(1):158-63

7. Trindade LL, Pires DEP. Implicações dos modelos assistenciais da atenção básica nas cargas de trabalho dos profissionais de saúde. Texto \& contexto enferm. 2013; 22(1):36-42.

8. Brasil. Portaria n. 2.436 de 21 de setembro de 2017. Aprova a Política Nacional de Atenção Básica, estabelecendo a revisão de diretrizes para a organização da Atenção Básica, no âmbito do Sistema Único de Saúde (SUS). Diário Oficial da União, 2017 set. 21 ; p. 68-76.

9. Resende MC, Azevedo EGS, Lourenço LR, Faria LS, Alves $\mathrm{NF}$, Farina NP, et al. Saúde mental e ansiedade em agentes comunitários que atuam em saúde da família em Uberlândia (MG, Brasil). Ciênc. saúde coletiva. 2011; 16(4):2115-2122.

10. Souza LJR, Freitas MCS. O agente comunitário de saúde: violência e sofrimento no trabalho a céu aberto. Rev. baiana saúde pública. 2011; 35(1):96-109.

11. Camelo SHH, Angerami ELS. Sintomas de estresse nos trabalhadores atuantes em cinco núcleos de saúde da família. Rev. latinoam. enferm. 2004; 12(1):14-21.

12. Paula IR, Marcacine PR, Castro SS, Walsh IAP. Capacidade para o trabalho, sintomas osteomusculares e qualidade de vida entre agentes comunitários de saúde em Uberaba. Saúde Soc. 2015; 24(1):152-64.

13. Brasil. Lei $n^{\circ} 8.080$ de 19 de setembro de 1990. Dispõe sobre as condições para a promoção, proteção e recuperação da saúde, a organização e o Funcionamento dos serviços correspondentes e dá outras providências. Diário Oficial da União. 1990 set. 20; p.18055.

14. Ferraz L, Aerts DRGC. O cotidiano de trabalho do agente comunitário de saúde no PSF em Porto Alegre. Ciênc. saúde coletiva. 2005; 10(2):347-55.

15. Bezerra AFB, Santo ACGE, Batista Filho M. Concepções e práticas do agente comunitário na atenção à saúde do idoso. Rev Saúde Pública. 2005; 39(5):809-15.

16. Brasil. Lei $n^{0} 13.595$ de 5 de janeiro de 2018. Altera a Lei $\mathrm{n}^{\circ} 11.350$, de 5 de outubro de 2006, para dispor sobre a reformulação das atribuições, a jornada e as condições de trabalho, o grau de formação profissional, os cursos de

\section{CONCLUSÃO}

Os ACS desse estudo apresentaram uma boa capacidade de trabalho de acordo com o ICT, entretanto, as doenças citadas por eles despontam como agravantes que podem vir a diminuir tal capacidade.

formação técnica e continuada e a indenização de transporte dos profissionais Agentes Comunitários de Saúde e Agentes de Combate às Endemias. Diário Oficial da União. 2018 jan. $8 ;$ p. 1.

17. Santos KTD, Saliba, NA, Moimaz SAS, Arcieri RM, Carvalho MDL. Agente comunitário de saúde: perfil adequado à realidade do Programa Saúde da Família? Ciênc. saúde coletiva. 2011; 16(Suppl.1):1023-28.

18. Gomes KO, Cotta RMM, Cherchiglia ML, Mitre SM, Batista RS. A práxis do agente comunitário de saúde no contexto do programa saúde da família: reflexões estratégicas. Saúde Soc. 2009; 18(4):744-55.

19. Vasconcellos NDPC, Costa-Val R. Avaliação da qualidade de vida dos agentes comunitários de saúde de Lagoa Santa-MG. Rev. APS. 2008. 11(1):17-28.

20. Mascarenhas CHM, Prado FO, Fernandes MH. Fatores associados à qualidade de vida de agentes comunitários de saúde. Ciênc. saúde coletiva. 2013; 18(5):1375-86.

21. Souza RC, Masson MLV, Araújo TM. Efeitos do exercício do trato vocal semiocluído em canudo comercial na voz do professor. Rev. CEFAC . 2017; 19(3):360-370.

22. Alcântara MA, Assunção AA. Influência da organização do trabalho sobre a prevalência de transtornos mentais comuns dos agentes comunitários de saúde de Belo Horizonte. Rev. bras. saude ocup. 2016; 41(e2):1-11.

23. Hilleshein EF, Lautert L. Capacidade de trabalhar, características sociodemográficas e trabalhistas de enfermeiros em um hospital universitário. Rev. latinoam. enferm. 2012; 20(3):T1-T8.

24. Magnago TSBS, Beck CLC, Greco PBT, Tavares JP, Prochnow A, Silva RM. Avaliação da capacidade para o trabalho dos trabalhadores de enfermagem de pronto-socorro. Rev. eletrônica enferm. 2013; 15(2):523-32.

25. Pereira IVS, Rocha MJL, Silva VM, Caldeira AP. Morbidade autorreferida por trabalhadores das Equipes de Saúde da Família. Ciênc. saúde coletiva. 2014; 19(2):461-468.

26. Ministério da Saúde (BR). Plano de ações estratégicas para o enfrentamento das doenças crônicas não transmissíveis (DCNT) no Brasil 2011-2022. Brasília: Ministério da Saúde; 2011.

27. Martinez MC, Latorre MRDO. Fatores associados à capacidade para o trabalho de trabalhadores do Setor Elétrico. Cad. saúde pública. 2009; 25(4):761-772.

28. Gomes MF, Lima ASR, Feitosa LS, Pontes Netto VB Nascimento RD, Andrade MS. Riscos e agravos ocupacionais: percepções dos agentes comunitários de saúde. Rev. pesqui. cuid. fundam. 2015; 7(4):3574-3586.

29. Viero NC, Greco PBT, Magnago TSBS, Scalcon CB, Prochnow $A$, Jacobi CS, et al. Redução da capacidade para o trabalho e a relação da morbidade dos trabalhadores de enfermagem. Revista Contexto \& Saúde. 2011; 10(20):1305-1310.

30. Barbosa REC, Assunção AA, Araújo TM. Distúrbios musculoesqueléticos em trabalhadores do setor saúde de Belo Horizonte, Minas Gerais, Brasil. Cad. saúde pública. 2012; 28(8):1569-1580

\section{CORRESPONDÊNCIA}

Andreia Marinho Barbosa

Rua José Dorian Pires Milfont, 40, Valentim Gonçalves

São João do Rio do Peixe - Paraíba - Brasil - CEP: 58910-000.

E-mail: amb_yeshua@yahoo.com.br 